\title{
Long-term X-ray variability of the microquasar system LS 5039/RX J1826.2-1450
}

\author{
P. Reig ${ }^{1}$, M. Ribó ${ }^{2}$, J. M. Paredes ${ }^{2, \star}$, and J. Marti ${ }^{3}$ \\ ${ }^{1}$ G.A.C.E., Institut de Ciències dels Materials, Universitat de Valencia, 46071 Paterna-Valencia, Spain \\ e-mail: pablo.reig@uv.es \\ 2 Departament d'Astronomia i Meteorologia, Universitat de Barcelona, Av. Diagonal 647, 08028 Barcelona, Spain \\ e-mail: mribo@am.ub.es; josep@am.ub.es \\ ${ }^{3}$ Departamento de Física, Escuela Politécnica Superior, Universidad de Jaén, Virgen de la Cabeza 2, 23071 Jaén, Spain \\ e-mail: jmarti@ujaen.es
}

Received 14 February 2003 / Accepted 16 April 2003

\begin{abstract}
We report on the results of the spectral and timing analysis of a BeppoSAX observation of the microquasar system LS 5039/RX J1826.2-1450. The source was found in a low-flux state with $F_{\mathrm{X}}(1-10 \mathrm{keV})=4.7 \times 10^{-12} \mathrm{erg} \mathrm{cm}^{-2} \mathrm{~s}^{-1}$, which represents almost one order of magnitude lower than a previous RXTE observation 2.5 years before. The $0.1-10 \mathrm{keV}$ spectrum is described by an absorbed power-law continuum with photon-number spectral index $\Gamma=1.8 \pm 0.2$ and hydrogen column density of $N_{\mathrm{H}}=1.0_{-0.3}^{+0.4} \times 10^{22} \mathrm{~cm}^{-2}$. According to the orbital parameters of the system, the BeppoSAX observation covers the time of an X-ray eclipse should one occur. However, the 1.6-10 keV light curve does not show evidence of such an event, which allows us to give an upper limit to the inclination of the system. The low X-ray flux detected during this observation is interpreted as a decrease in the mass accretion rate onto the compact object due to a decrease in the mass-loss rate from the primary.
\end{abstract}

Key words. stars: individual: LS 5039, RX J1826.2-1450, 3EG J1824-1514 - X-rays: stars - stars: variables: general radio continuum: stars

\section{Introduction}

LS 5039/RX J1826.2-1450 was first identified as a new massive X-ray binary by Motch et al. (1997). Interest in this source has grown significantly because it has turned out to be a source of persistent radio-emitting relativistic jets, and because it is believed to be physically associated with the $\gamma$-ray source 3EG J1824-1514 (Paredes et al. 2000, 2002).

The first radio detection was reported by Martí et al. (1998) using the Very Large Array, but the discovery of radio jets was only possible when the source was observed at milliarcsecond scales with the Very Long Baseline Array (Paredes et al. 2000). The radio emission is persistent, non-thermal and variable, but no strong radio outbursts or periodic variability have been detected (Ribó et al. 1999, hereafter R99; Ribó 2002).

In the optical band LS 5039/RX J1826.2-1450 appears as a bright $V=11.2,06.5 \mathrm{~V}((\mathrm{f}))$ star showing little variability on timescales of months to years (Clark et al. 2001). Variations of $\sim 0.4$ mag have been reported in the infrared ( $H$ and $K$ bands) but no obvious mechanisms for such variability have been proposed (Clark et al. 2001). Recent studies show that LS 5039

Send offprint requests to: P. Reig, e-mail: pablo.reig@uv.es

* CER on Astrophysics, Particle Physics and Cosmology. Universitat de Barcelona. is a runaway system moving away from the Galactic plane with a total systemic velocity of $\sim 150 \mathrm{~km} \mathrm{~s}^{-1}$ and a component perpendicular to the Galactic plane larger than $100 \mathrm{~km} \mathrm{~s}^{-1}$ (Ribó et al. 2002; McSwain \& Gies 2002). The orbit of LS 5039/RX J1826.2-1450 was first studied by McSwain et al. (2001), who found that LS 5039/RX J1826.2-1450 is a shortperiod, $P_{\text {orb }}=4.117 \pm 0.011$ day and highly eccentric, $e=$ $0.41 \pm 0.05$, system. Spectroscopic observations carried out in July 2002 by Casares et al. (2003) seem to confirm the orbital period of the system, but neither confirm nor reject the proposed eccentricity.

In the X-rays LS 5039/RX J1826.2-1450 has been studied by R99 using RXTE data. The X-ray timing analysis indicates the absence of pulsed or periodic emission on time scales of $0.02-2000 \mathrm{~s}$. The source spectrum is well represented by a power-law model, plus a Gaussian component describing a strong iron line at $6.6 \mathrm{keV}$. Significant emission is seen up to $30 \mathrm{keV}$, and no exponential cut-off at high energy is required. The X-ray luminosity has been shown to be compatible with accretion from the stellar wind of the optical companion (McSwain \& Gies 2002).

One of the open questions that remains to be solved is the nature of the compact object of the system as no conclusive data exists. The absence of X-ray pulsations and 


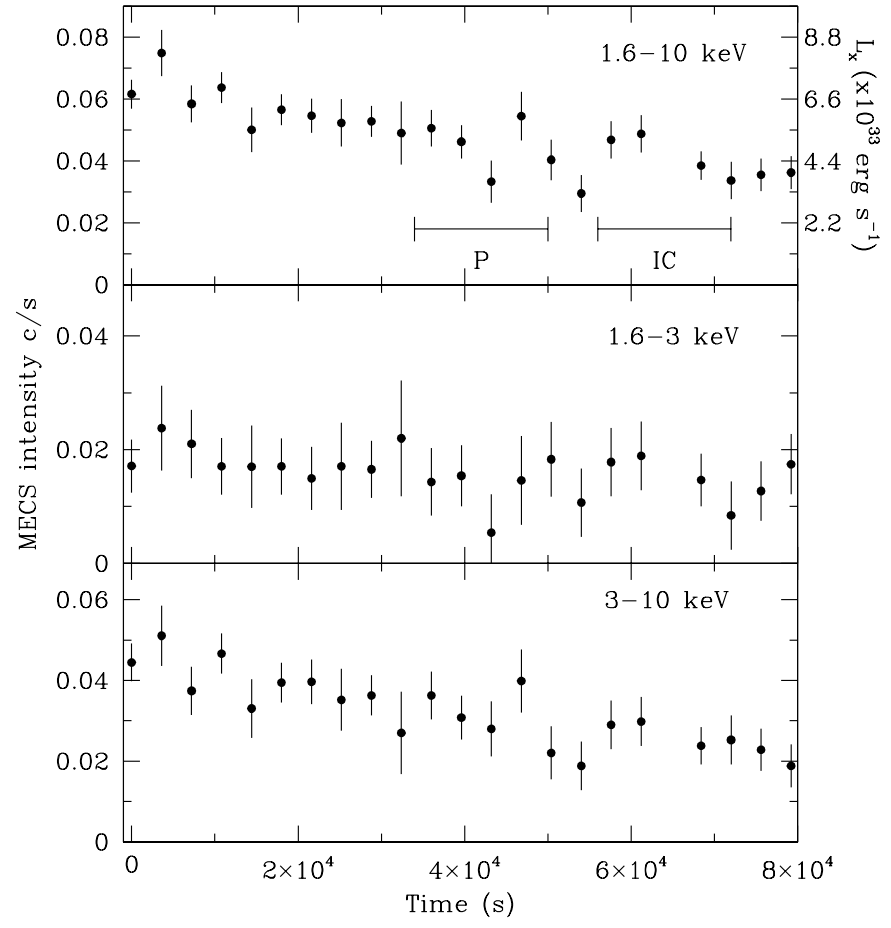

Fig. 1. MECS background subtracted light curves of RX J1826.2-1450 in different energy ranges. Each point represents $60 \mathrm{~min}$. Time zero corresponds to JD 2451825.75 . The expected time of periastron (P) and inferior conjunction (IC) of the primary are indicated in the top panel, where the error bars represent the uncertainty in the zero point of the ephemeris. The time difference between these two points is $2.2 \times 10^{4} \mathrm{~s}$. An estimate of the unabsorbed $\mathrm{X}$-ray luminosity according to the model of Table 1 is also given in the right axis of the top panel.

exponential cut-off at $\sim 30 \mathrm{keV}$ and the persistent non-thermal emission favours the black-hole classification (R99). On the other hand, the value of the mass function of the system $f(m)=$ $0.00103 M_{\odot}(\mathrm{McSwain}$ et al. 2001) and the characteristics of its accretion mechanism (McSwain \& Gies 2002) appear to be more consistent with a neutron star companion.

In this work we present an analysis of the X-ray timing and spectroscopic properties of RX J1826.2-1450 using the narrow field instruments on board the BeppoSAX satellite. This observation permits us to derive a more accurate value of the absorption to the source and study its long-term X-ray variability. We also present optical spectroscopic observations around the $\mathrm{H} \alpha$ line carried out at the Skinakas Observatory.

\section{Observations and results}

\subsection{X-ray observations}

LS 5039/RX J1826.2-1450 was observed by BeppoSAX on October 8, 2000 (JD 2451825.79-2451826.71) for about $80 \mathrm{ks}$. BeppoSAX (Boella et al. 1997a) carried four narrow field instruments (LECS, MECS, HPGSPC and PDS) and a wide field camera (WFC). Here we report on the results from the lower energy detectors: the Low-Energy (LECS) and MediumEnergy (MECS) Concentrator Spectrometers. They consisted of a set of four and three X-ray concentrators respectively

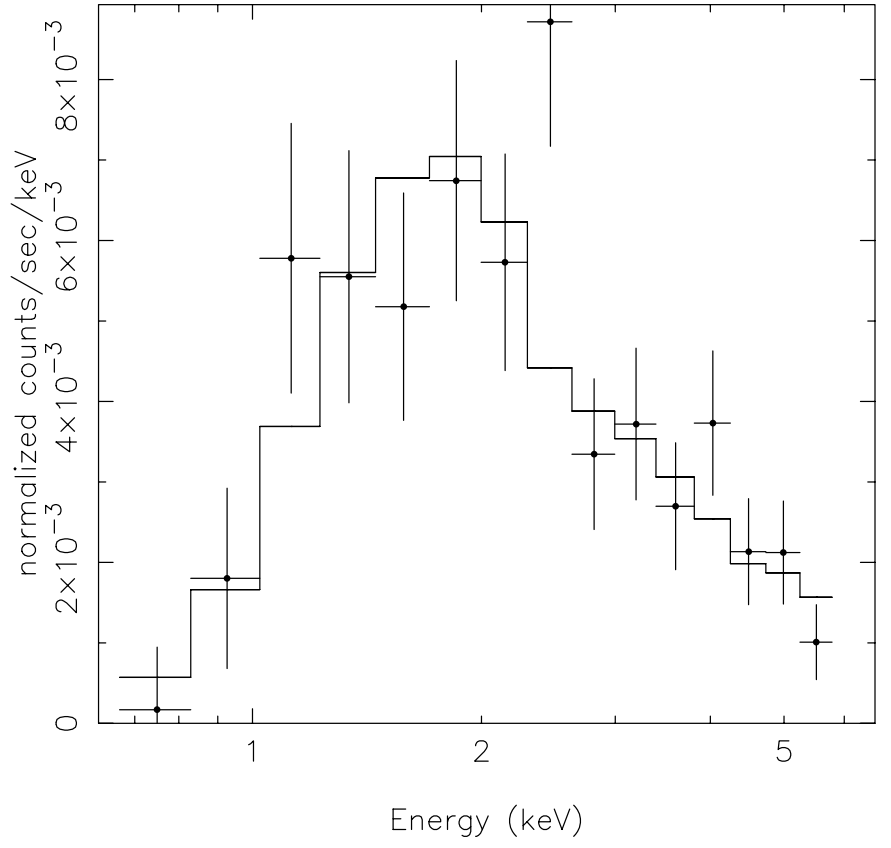

Fig. 2. LECS energy spectrum of RX J1826.2-1450 and the best-fit model displayed in Table 1.

together with imaging gas scintillation proportional counter detectors located at the focal planes. In the LECS (Parmar et al. 1997), one of these detectors was sensitive to X-rays in the energy range $0.1-10 \mathrm{keV}$, while the other three covered an energy range of 1.3-10 keV. The MECS (Boella et al. 1997b) operated in the energy range 1.6-10 $\mathrm{keV}$. Each detector unit provided a total collecting (geometrical) area of $\sim 124 \mathrm{~cm}^{2}$, an energy resolution of $<8.8 \%$ at $6 \mathrm{keV}$ and a maximum time resolution of $16 \mu \mathrm{s}$. Note that one of the MECS became inoperative on May 9, 1997 due to a fault in the unit's gas cell high voltage supply.

Light curves and spectra were extracted from circular regions with a radius of $4^{\prime}$ for both the LECS and the MECS instruments. We selected two source-free regions (of radius $4^{\prime}$ each) in the field of view, and obtained a mean light curve in order to account for the instrumental background. For the spectral analysis, the background was obtained from a blank field using a region similar in size and position to the source extraction region.

The X-ray intensity in the energy range $1.6-10 \mathrm{keV}$ decreased with time from a value of $\sim 0.06$ MECS count $\mathrm{s}^{-1}$ at the beginning of the observation to $\sim 0.03$ MECS count $\mathrm{s}^{-1}$ at the end (Fig. 1). A fit to a constant gave an unacceptable reduced $\chi^{2}(>2)$. However, adding a linear term with negative slope we obtained a reduced $\chi^{2} \sim 1$. An F-test gave a probability of less than $0.7 \%$ that this improvement in the quality of the fit could occur by chance. The flux variability occurs at higher energies. Below $\sim 3 \mathrm{keV}$ the flux remains fairly constant (Fig. 1).

Acceptable fits of the X-ray energy spectra were obtained with an absorbed power-law model for both LECS (Fig. 2) and MECS (Fig. 3). Table 1 shows the best-fit spectral parameters. The power law was modified by a low-energy 


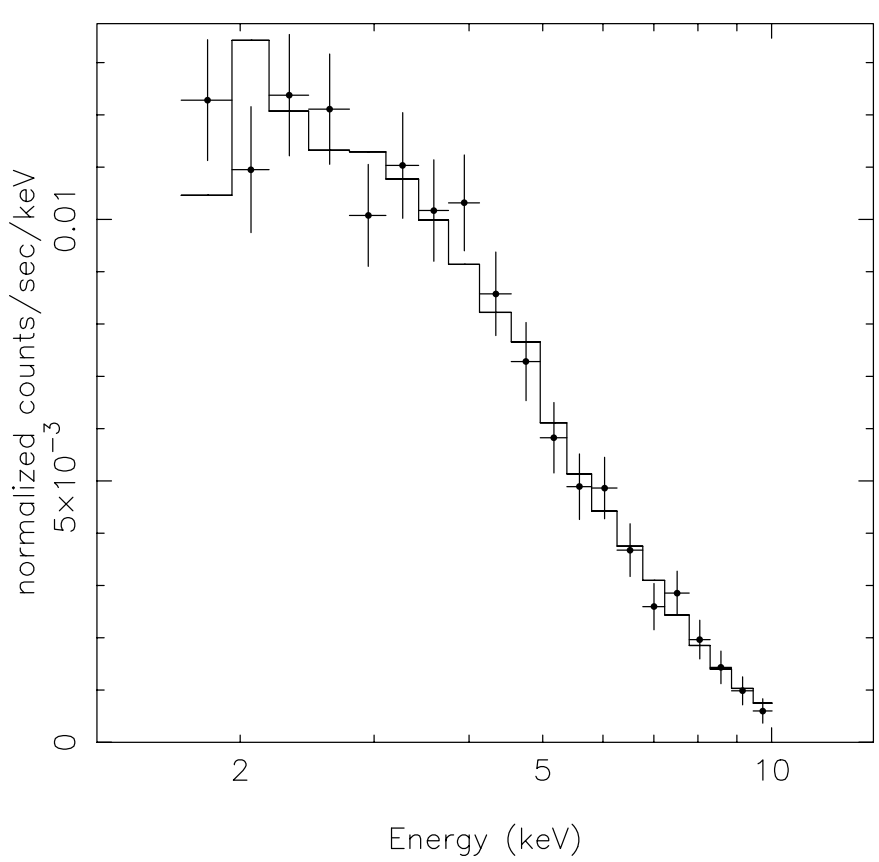

Fig. 3. MECS energy spectrum of RX J1826.2-1450 and the best-fit model displayed in Table 1.

absorption component of the form $e^{-N_{\mathrm{H}} \sigma(E)}$, where $N_{\mathrm{H}}$ is the equivalent hydrogen column density and $\sigma(E)$ the photoelectric cross-section of Morrison \& McCammon (1983). The powerlaw index and the hydrogen column density have values of $\sim 1.8$ and $\sim 1.0 \times 10^{22} \mathrm{~cm}^{-2}$, respectively (see discussion in Sect. 3.1). The LECS (0.1-5 keV) and MECS (1.6-10 keV) absorbed Xray luminosity was $1.9 \times 10^{33} \mathrm{erg} \mathrm{s}^{-1}$ and $4.6 \times 10^{33} \mathrm{erg} \mathrm{s}^{-1}$, respectively, assuming a distance of 2.9 kpc (Ribó et al. 2002).

\subsection{Optical observations}

Optical spectroscopic observations were made with the $1.3 \mathrm{~m}$ telescope of the Skinakas Observatory in Crete (Greece) on the night of July 26, 1999 and September 10, 2002. The instrumental set-up consisted of a $2000 \times 800$ ISA SITe CCD and a $13021 \mathrm{~mm}^{-1}$ grating, giving a dispersion of $\sim 1 \AA /$ pixel. The reduction of the spectra was made using the STARLINK Figaro package (Shortridge et al. 2001), while their analysis was performed using the STARLINK Dipso package (Howarth et al. 1998).

The wavelength coverage of the observations included the $\mathrm{H} \alpha$ line $(\lambda 6563 \AA)$. The strength of the stellar wind emission in $\mathrm{H} \alpha$ provides information about the mass-loss rate in O-type stars (Puls et al. 1996 and references therein). The measurement of the equivalent width of the $\mathrm{H} \alpha$ line $(E W(\mathrm{H} \alpha))$ in LS 5039 is hampered by the presence of other stellar lines, especially He II $\lambda 6527$. Nevertheless, when the emission is low the main factor contributing to the uncertainty in the equivalent width is the difficulty in obtaining the intensity of the continuum. In Table 2 we give the values of $E W(\mathrm{H} \alpha)$ as contemporaneous as possible with the X-ray measurements.
Table 1. Spectral fit results for the absorbed power-law model. Uncertainties are given $68 \%$ confidence for one parameter of interest.

\begin{tabular}{ll}
\hline \hline Parameters & Best-fit values \\
\hline \multicolumn{1}{c}{ LECS } \\
\hline$N_{\mathrm{H}}\left(\mathrm{cm}^{-2}\right)$ & $1.0_{-0.3}^{+0.4} \times 10^{22}$ \\
$\Gamma$ & $1.8 \pm 0.3$ \\
$F_{\mathrm{X}}(0.1-5 \mathrm{keV})\left(\mathrm{erg} \mathrm{cm}^{-2} \mathrm{~s}^{-1}\right)$ & $1.9 \times 10^{-12}$ \\
$\chi_{\mathrm{r}}^{2}($ d.o.f. $)$ & $1.25(12)$ \\
\hline \multicolumn{2}{c}{$\mathrm{MECS}$} \\
\hline$N_{\mathrm{H}}\left(\mathrm{cm}^{-2}\right)$ & $1.2 \pm 0.4 \times 10^{22}$ \\
$\Gamma$ & $1.8 \pm 0.1$ \\
$F_{\mathrm{X}}(1.6-10 \mathrm{keV})\left(\mathrm{erg} \mathrm{cm}^{-2} \mathrm{~s}^{-1}\right)$ & $4.6 \times 10^{-12}$ \\
$\chi_{\mathrm{r}}^{2}($ d.o.f. $)$ & $0.87(17)$ \\
\hline
\end{tabular}

\section{Discussion}

\subsection{Hydrogen column density}

The RXTE observation reported by R99 was not able to provide a reliable value of the absorption to the source due to the insensitivity of the detectors to X-rays below $2.5 \mathrm{keV}$, where most of the interstellar absorption of X-ray photons takes place. The sensitivity of the BeppoSAX narrow field instruments to X-rays below $2 \mathrm{keV}$, especially LECS, allows us to derive a more accurate value of the hydrogen column density, $N_{\mathrm{H}}=1.0_{-0.3}^{+0.4} \times 10^{22} \mathrm{~cm}^{-2}$. Assuming $E(B-V)=A_{V} / 3.1$ (Rieke \& Lebofsky 1985) the X-ray absorption can be converted into optical extinction by means of the empirical relations of Predehl \& Schmitt (1995), to obtain $E(B-V)=1.8_{-0.5}^{+0.7}$, or those of Ryter et al. (1975) and Gorenstein (1975), to obtain $E(B-V)=1.5_{-0.4}^{+0.6}$. These values of $E(B-V)$ are considerably higher than the unreliable value of $E(B-V) \approx 0.4$ derived from the PCA/RXTE observation (R99).

On the other hand, optical photometry of LS 5039 can be found in Drilling (1991) and Lahulla \& Hilton (1992) who gave a value for the colour $(B-V)=0.95 \pm 0.01$ and Clark et al. (2001), who reported $(B-V)=0.85 \pm 0.02$. Using the average of these values and assuming an intrinsic colour index of $(B-V)_{0}=-0.30 \pm 0.02$ for an O6.5V star (Lejeune \& Scharer 2001) we obtain a colour excess of $E(B-V)=1.2 \pm 0.1$. The extinction obtained from the BeppoSAX observation is somehow higher than (although statistically consistent with) that derived from the optical observations. One explanation for this difference might be the fact that the hydrogen column density is more sensitive to absorption by both interstellar and intrinsic (i.e. in the vicinity of the X-ray source) matter, while the optical indicators would mainly account for the interstellar absorption. The presence of significant amounts of cold matter in the surroundings of the system is supported by the detection of the relatively strong iron (equivalent width of $0.75 \pm$ $0.06 \mathrm{keV}$ ) emission line at $6.6 \mathrm{keV}$ in the 3-30 keV X-ray spectrum of LS 5039/RX J1826.2-1450 (R99). Unfortunately, due to limited signal-to-noise ratio no line is detected in the BeppoSAX data. 


\subsection{Absence of $X$-ray eclipse}

Using the ephemeris of McSwain et al. (2001), the BeppoSAX observation covers the orbital phase from 0.89 to 0.11 . Note that this observation took place just four days after the periastron date used by these authors in their ephemeris, i.e., JD $2451822.12 \pm 0.09$. Consequently, our determination of the orbital phase is not strongly affected by the lack of accuracy in the value of the orbital period ( $P_{\text {orb }}=4.117 \pm 0.011$ days). Hence, periastron took place on JD $2451826.24 \pm 0.09$, or at $4.2 \pm 0.8 \times 10^{4} \mathrm{~s}$ in Fig. 1. The epoch of inferior conjunction of the optical star, which would correspond to the time of an X-ray eclipse, is 0.25 days after periastron or at orbital phase 0.06 (McSwain et al. 2001), which corresponds to JD $2451826.49 \pm 0.09$, or to $6.4 \pm 0.8 \times 10^{4} \mathrm{~s}$ in Fig. 1 . The duration of a possible eclipse would last less than 0.2 days $\left(1.7 \times 10^{4} \mathrm{~s}\right)$ for $M_{\mathrm{opt}}=40 M_{\odot}, R_{\mathrm{opt}}=10 R_{\odot}$ and $i=90^{\circ}$ (shorter for a lower inclination and an eventual lower mass for the primary) and hence it should have been detected by BeppoSAX.

Although the light curve does not show statistical significant evidence for an eclipse, the variability increases between periastron and inferior conjunction, possibly indicating some kind of distortion of the stellar wind when the compact star finds itself at minimum distance from the primary. There is also a trend for the count rate to decrease as the source approaches inferior conjunction. This trend is detected at energies above $3 \mathrm{keV}$, but not at lower energies, contrary to what would be expected if this was due to a change in absorption, as it happens in Cygnus X-1 (Wen et al. 1999). Therefore, this decrease in the hard X-ray flux during the BeppoSAX observation is probably intrinsic, and may reflect changes in the accretion rate due to orbital motion.

The lack of eclipses allows us to set an upper limit on the inclination of the system $i$. This implies, in the case of a spherical companion (no photometric ellipsoidal modulation has been found to date), that $\cos i>R_{\mathrm{opt}} / r$, where $R_{\mathrm{opt}}$ is the radius of the optical companion an $r$ is its distance to the compact object at inferior conjunction (phase 0.06). Assuming a mass of $40 M_{\odot}$ and a radius of $10 R_{\odot}$ for an $06.5 \mathrm{~V}$ star (Howarth \& Prinja 1989), the canonical mass for a neutron star $\left(1.4 M_{\odot}\right)$ and the orbital parameters of McSwain et al. (2001), $e=0.41 \pm 0.05$ and $P_{\text {orb }}=4.117 \pm 0.011$ days, we obtain an upper limit for the inclination of the system of $i<66 \pm 2^{\circ}$, where the upper and lower values correspond to the range of eccentricities given above. This inclination is consistent with the one, $i_{f}$, derived from the mass function, $f(m)=0.00103 \pm 0.00020 M_{\odot}$ (McSwain et al. 2001), for the same parameters, namely, $i_{f}=60^{\circ}$.

Note that OB stars in X-ray binary systems may be less massive than the corresponding field stars of the same spectral type (Kaper 2001). A decrease of the mass of the optical companion by about $10 \%$ reduces $i_{f}$ to $54^{\circ}$, while the upper limit hardly changes. On the other hand, assuming $M_{\mathrm{opt}}=40 M_{\odot}$ and the above mentioned orbital parameters Ribó et al. (2002) derived an upper limit for the mass of the compact object of $9 M_{\odot}$ (in order to avoid breackup speed of the primary once $V_{\text {rot }} \sin i$ is known). For a $9 M_{\odot}$ black-hole companion a
Table 2. X-ray flux and $\mathrm{H} \alpha$ equivalent width measurements of LS 5039. Errors in $E W(\mathrm{H} \alpha)$ are $\lesssim 10 \%$.

\begin{tabular}{lcc|cl}
\hline \hline Mission & $\begin{array}{l}\text { Date } \\
\text { X-ray obs. }\end{array}$ & $F_{\mathrm{X}}^{a}$ & $\begin{array}{c}E W(\mathrm{H} \alpha) \\
(\AA)\end{array}$ & $\begin{array}{l}\text { Date } \\
\text { Opt. obs. }\end{array}$ \\
\hline RXTE & $8 / 02 / 1998$ & $40^{b}$ & $2.2^{c}$ & Aug. 1998 \\
ASCA & $4 / 10 / 1999$ & $10.9^{b}$ & $2.8^{b}$ & $26 / 7 / 1999$ \\
BeppoSAX & $8 / 10 / 2000$ & $4.9^{b}$ & $3.1^{c}$ & Oct. 2000 \\
CHANDRA & $10 / 09 / 2002$ & $10.0^{d}$ & $2.8^{b}$ & $10 / 9 / 2002$ \\
\hline
\end{tabular}

${ }^{a} \times 10^{-12} \mathrm{erg} \mathrm{cm}^{-2} \mathrm{~s}^{-1}$ in the energy range $0.3-10 \mathrm{keV}$.

$b$ This work.

c McSwain \& Gies (2002).

$d$ Miller (private communication).

similar analysis yields $i<68 \pm 2^{\circ}$ and $i_{f}=8.5^{\circ}$. Similar values are obtained if we decrease the mass of the optical companion by about $10 \%$. In either case, the lack of X-ray eclipses constrains the inclination of the system to values smaller than $70^{\circ}$, which is clearly compatible with those found using the mass function of the system.

\subsection{Long-term $X$-ray variability}

In order to study the long-term X-ray variability of LS 5039/RX J1826.2-1450 we obtained the X-ray luminosity in the same energy ranges as given in previous missions. The value of the ROSAT 1996 October 0.1-2.4 keV (Motch et al. 1997) and the RXTE 1998 February 3-30 keV (R99) X-ray luminosities are $7.1 \times 10^{33} \mathrm{erg} \mathrm{s}^{-1}$ and $5.2 \times 10^{34} \mathrm{erg} \mathrm{s}^{-1}$, respectively. The $0.1-2.4 \mathrm{keV}$ X-ray luminosity obtained with LECS is $6.6 \times 10^{32} \mathrm{erg} \mathrm{s}^{-1}$ and that obtained with MECS in the energy range $3-30 \mathrm{keV}$ is $8.0 \times 10^{33} \mathrm{erg} \mathrm{s}^{-1}$ (derived assuming the model given in Table 1). This observation took place in 2000 October. All values are for an assumed distance of $2.9 \mathrm{kpc}$ (Ribó et al. 2002). Clearly the source was in a low-flux state during the BeppoSAX observation (about one order of magnitude fainter) when compared to the epoch of the ROSAT and RXTE observations. As explained above this low flux, however, cannot be accounted for by invoking a partial X-ray eclipse. We have also inspected archival X-ray data and found an ASCA observation of LS 5039/RX J1826.2-1450 made on 1999 October 4. We have obtained the X-ray flux in the energy range $0.3-10 \mathrm{keV}$, shown in Table 2, which is between the RXTE and BeppoSAX measurements. In addition, a similar $\mathrm{X}$-ray flux has been recently detected with CHANDRA (Miller, private communication), and is also reported in Table 2.

We also studied the long-term optical variability of the primary by measuring the strength of the $\mathrm{H} \alpha$ line. Table 2 shows the values of the $\mathrm{H} \alpha$ equivalent width together with the X-ray flux from the missions that have observed LS 5039/RX J1826.2-1450. The $\mathrm{H} \alpha$ emission was weakest during the BeppoSAX observation in October 2000 and strongest a few months after the RXTE pointing in February 1998, when the X-ray emission was also strong (since the line is in absorption, the smaller $E W(\mathrm{H} \alpha)$ the stronger the emission). Moreover, very similar $E W(\mathrm{H} \alpha)$ are found contemporaneous to the ASCA and CHANDRA observations, as the 
detected X-ray fluxes are, and also between the $E W(\mathrm{H} \alpha)$ measurements contemporaneous to the RXTE and BeppoSAX observations. Hence, there is a trend in the sense that the lower the emission in $\mathrm{H} \alpha$, the lower the observed X-ray flux. All these results suggest that a decrease in the mass-loss rate from the primary induces a decrease in the emitted X-ray flux of the binary system. In this context, we propose that changes in the mass-loss rate from the primary, reflected in the change of $E W(\mathrm{H} \alpha)$, can account for a change in the mass accretion rate onto the compact object, and hence explain the detected changes in X-ray luminosity.

On the other hand, since this binary system has a high eccentricity, we cannot rule out a priori that the detected variations in the X-ray flux are not due to orbital variability. If the mass transfer in LS 5039/RX J1826.2-1450 occurs via the strong stellar wind of the optical primary then, given the high eccentricity of the system, some variations in the X-ray luminosity with the orbital phase should be expected. The fraction of the stellar wind captured by the compact object depends on its velocity relative to the wind. Also, variations in the density of the wind may produce changes in the amount of absorption material throughout the orbit giving rise to the X-ray flux variations. Orbital flux variations have been seen in many high-mass X-ray binaries powered by stellar wind accretion: 4U 1700-37 (Haberl \& Day 1992), GX 301-2 (Haberl 1991), Vela X-1 (Haberl \& White 1990), 4U 1907+09 (Marshall \& Ricketts 1980). With its small orbit and early-type optical primary LS 5039/RX J1826.2-1450 is expected to share many similarities with these systems.

However, a purely Bondi-Hoyle accretion model (Bondi 1952) does not reproduce the observations. Figure 4 shows the expected variability of the X-ray luminosity in LS 5039/RX J1826.2-1450 using a spherically symmetrical Bondi-Hoyle accretion model. For each orbital phase and using the formulae given in the Appendix, we computed the binary separation, the orbital and wind velocities, the relative velocity, the wind density, the accretion rate and finally the X-ray luminosity. We note that this is a bolometric unabsorbed X-ray luminosity, to be compared to the much lower observed luminosity in the range 1.6-10 keV (top panel of Fig. 1). Although this discrepancy could also be caused by the lack of precise knowledge of the parameter values used in the model, there are two strong arguments against this model. First, we should expect a variation in flux of at least a factor of 2 within the phase interval of the BeppoSAX observations as follows: starting with a gradual rise, peaking smoothly around phase $0.02\left(\sim 5 \times 10^{4} \mathrm{~s}\right.$ in Fig. 1) and ending with a gradual decrease. Second, since the BeppoSAX observation took place during periastron the X-ray luminosity should be the largest, contrary to what it is observed (see Table 2).

Although it is clear that detailed observations covering a full orbital period are needed in order to better study and model the orbital X-ray variability of LS 5039, it seems unlikely that it can be responsible for the variations of one order of magnitude quoted in Table 2 . Therefore, we conclude that this variability may be due to changes in the stellar wind mass-loss rate on longer timescales.

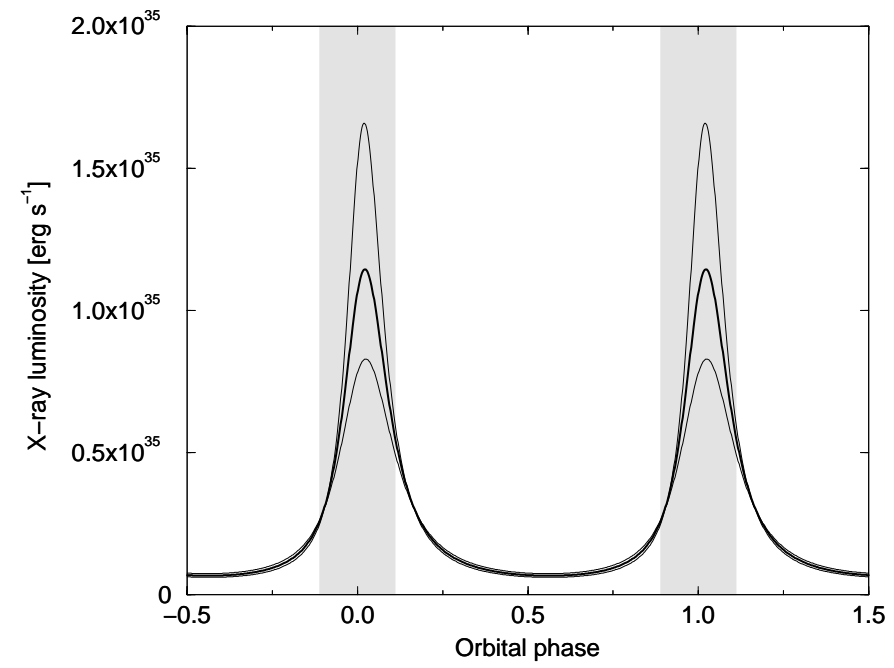

Fig. 4. Expected variability of the X-ray luminosity in LS 5039 due to the significantly eccentric orbit of the compact companion around the $06.5 \mathrm{~V}((\mathrm{f}))$ star with a period $P_{\text {orb }}=4.117 \mathrm{~d}$, using a spherically symmetrical Bondi-Hoyle accretion model and the parameters quoted in the text. The thick line corresponds to $e=0.41$, the nominal eccentricity value in the McSwain et al. (2001) solution, while the lower and upper thin lines are for $e=0.36$ and $e=0.46$, which correspond to the error limits quoted by the authors. Phase 0 corresponds to periastron, while the shaded areas correspond to the phase interval covered by our BeppoSAX observation.

\section{Conclusions}

The main conclusions of the analysis of our BeppoSAX observation of the microquasar LS 5039/RX J1826.2-1450 can be summarized as follows:

1. We have obtained the most accurate value of the hydrogen column density to date: $N_{\mathrm{H}}=1.0_{-0.3}^{+0.4} \times 10^{22} \mathrm{~cm}^{-2}$. The derived colour excess is compatible but higher than the one obtained from optical photometry alone, suggesting that there might be intrinsic absorption within the binary system orbit.

2. Our observation took place near inferior conjunction, and no X-ray eclipse has been detected. This fact has allowed us to compute upper limits for the inclination of the binary system for different masses of the compact object: $i<66^{\circ}$ for $M_{\mathrm{X}}=1.4 M_{\odot}$ and $i<68^{\circ}$ for $M_{\mathrm{X}}=9 M_{\odot}$. These values are compatible with the ones obtained using the mass function.

3. We have found hints of a correlation between the measured $E W(\mathrm{H} \alpha)$ and the observed X-ray flux on timescales of years, in the sense that the stronger the optical emission the higher the X-ray flux. In this context, we have suggested that a decrease in the mass-loss rate from the primary induces a decrease in the mass accretion rate onto the compact object, and hence a decrease in the observed Xray flux.

4. A simple Bondi-Hoyle accretion model (without taking into account a varying absorption) cannot explain the X-ray variability because it predicts an increase in the X-ray flux near periastron that is not observed. This may happen because the model is very sensitive to some parameters not 
yet very well constrained, like the eccentricity and the wind velocity. However, the fact that no significant variability is detected near periastron suggests that the observed variability in the X-ray flux on timescales of years is due to changes in the mass-loss rate of the primary. An X-ray monitoring during a full $\sim 4 \mathrm{~d}$ orbital cycle would clarify these ideas.

\section{Appendix: Orbital Bondi-Hoyle X-ray variability}

An order of magnitude estimate of the orbital X-ray variability can be obtained using the Bondi-Hoyle accretion model. The accretion luminosity is then computed by means of the simple formula:

$L_{\mathrm{X}} \simeq \frac{G M_{\mathrm{X}} \dot{M}}{R_{\mathrm{X}}}$

where $G$ is the gravitational constant, $M_{\mathrm{X}}$ and $R_{\mathrm{X}}$ the mass and radius of the compact object and $\dot{M}$ its variable accretion rate. Assuming a neutron star, we will proceed adopting $M_{\mathrm{X}}=1.4 M_{\odot}$ and $R_{\mathrm{X}}=10 \mathrm{~km}$. If the wind accretion onto the compact companion can be described as a spherical inflow (Bondi 1952), the accretion rate as a function of the binary separation $r$ is given by:

$\dot{M}=4 \pi\left(G M_{\mathrm{X}}\right)^{2} \frac{v_{\mathrm{w}}(r) \rho(r)}{v_{\text {rel }}^{4}(r)}$,

where $v_{\mathrm{w}}(r)$ is the wind velocity, $\rho(r)$ is the density of the stellar wind and $v_{\text {rel }}(r)$ its supersonical velocity relative to the neutron star. We will approximate the wind velocity $v_{\mathrm{w}}(r)$ by a typical $\beta$-law appropriate for O-type stars:

$v_{\mathrm{w}}(r)=v_{\infty}\left[1-\frac{R_{\mathrm{opt}}}{r}\right]^{\beta}$.

Here, $v_{\infty}$ is the wind velocity at infinity and $\beta$ a power index. Assuming a simple radial flow, the corresponding wind density can be derived from the continuity equation as:

$\rho(r)=\frac{\dot{M}_{\mathrm{opt}}}{4 \pi r^{2} v_{\mathrm{w}}(r)}$

Following McSwain \& Gies (2002), suitable values for LS 5039 are $v_{\infty}=2850 \mathrm{~km} \mathrm{~s}^{-1}, \beta=0.8$ and $\dot{M}_{\text {opt }}=8 \times$ $10^{-7} M_{\odot} \mathrm{yr}^{-1}$. To proceed, we have solved numerically the Keplerian motion of the neutron star around the $M_{\text {opt }}=40 M_{\odot}$ companion calculating both the eccentric and true anomalies $(E$ and $V$ ). The relative velocity between the wind and the neutron star is then given by:

$$
\begin{aligned}
v_{\mathrm{rel}}^{2}(r)= & {\left[\frac{n a^{2}}{r} \sin E+v_{\mathrm{w}}(r) \cos V\right]^{2} } \\
& +\left[\frac{n a^{2} \sqrt{1-e^{2}}}{r} \cos E-v_{\mathrm{w}}(r) \sin V\right]^{2},
\end{aligned}
$$

where $a$ is the semimajor axis and $n=2 \pi / P_{\text {orb }}$ is the mean angular motion.
Acknowledgements. We are grateful to J. Miller for permission to include in this paper unpublished results from his CHANDRA observation. We are also grateful for useful comments and suggestions from the anonymous referee. P.R. is a researcher of the programme Ramón y Cajal funded by the University of Valencia and Spanish Ministery of Science and Technology. M.R., J.M.P. and J.M. acknowledge partial support by DGI of the Ministerio de Ciencia y Tecnología (Spain) under grant AYA2001-3092, as well as partial support by the European Regional Development Fund (ERDF/FEDER). During this work, M.R. has been supported by a fellowship from CIRIT (Generalitat de Catalunya, ref. 1999 FI 00199). J.M. has been aided in this work by an Henri Chrétien International Research Grant administered by the American Astronomical Society, and has been partially supported by the Plan Andaluz de Investigación of the Junta de Andalucía (ref. FQM322). Skinakas Observatory is a collaborative project of the University of Crete, the Foundation for Research and Technology-Hellas and the Max-Planck-Institut für Extraterrestrische Physik.

\section{References}

Boella, G., Butler, R. C., Perola, G. C., et al. 1997a, A\&AS, 122, 299 Boella, G., Chiappetti, L., Conti, G., et al. 1997b, A\&AS, 122, 327

Bondi, H. 1952, MNRAS, 112, 195

Casares, J., Ribó, M., Paredes, J. M., \& Martí, J. 2003, in preparation Clark, J. S., Reig, P., Goodwin, S. P., et al. 2001, A\&A, 376, 476

Drilling, J. S. 1991, ApJS, 76, 1033

Gorenstein, P. 1975, ApJ, 198, 95

Haberl, F., \& White, N. E. 1990, ApJ, 361, 225

Haberl, F. 1991, ApJ, 376, 245

Haberl, F., \& Day, C. S. R. 1992, A\&A, 263, 241

Howarth, I. D., \& Prinja, R. K. 1989, ApJS, 69, 527

Howarth, I. D., Murray, J., Mills, D., \& Berry, D. S. 1998, Starlink User Note, 50.21

Kaper, L. 2001, in The influence of binaries on stellar population studies, ed. D. Vanbeveren, \& W. van Rensbergen, ASSL, 264, $125 \mathrm{r}, 15,65$

Lahulla, J. F., \& Hilton, J. 1992, A\&AS, 94, 265

Lejeune, T., \& Schaerer, D. 2001, A\&A, 366, 538

Marshall, N., \& Ricketts, M. J. 1980, MNRAS, 193, 7

Martí, J., Paredes, J. M., \& Ribó, M. 1998, A\&A, 338, L71

McSwain, M. V., \& Gies, D. R. 2002, ApJ, 568, L27

McSwain, M. V., Gies, D. R., Riddle, R. L., Wang, Z., \& Wingert, D. W. 2001, ApJ, 558, L43

Morrison, D., \& McCammon, D. 1983, ApJ, 270, 119

Motch, C., Haberl, F., Dennerl, K., Pakull, M., \& Janot-Pacheco, E. 1997, A\&A, 323, 853

Paredes, J. M., Martí, J., Ribó, M., \& Massi, M. 2000, Science, 288, 2340

Paredes, J. M., Ribó, M., Ros, E., Martí, J., \& Massi, M. 2002, A\&A, 393, L99

Parmar, A. N., Martin, D. D. E., Bavdaz, M., et al. 1997, A\&AS, 122, 309

Predehl, P., \& Schmitt, J. H. M. M. 1995, A\&A, 293, 889

Puls, J., Kudritzki, R.-P., Herrero, A., et al. 1996, A\&A, 305, 171

Ribó, M. 2002, Ph.D. Thesis, Universitat de Barcelona

Ribó, M., Reig, P., Martí, J., \& Paredes, J. M. 1999, A\&A, 347, 518 (R99)

Ribó, M., Paredes, J. M., Romero, G. E., et al. 2002, A\&A, 384, 954

Rieke, G. H., \& Lebofsky, M. J. 1985, ApJ, 288, 618

Ryter, C., Cesarsky, C. J., \& Audouze, J. 1975, ApJ, 198, 103

Shortridge, K., Meyerdierks, H., Currie, M., et al. 2001, Starlink User Note, 86.19

Wen, L., Cui, W., Levine, A. M., \& Bradt, H. V. 1999, ApJ, 525, 968 